\title{
Diversity and Novelty in Web Search, Recommender Systems and Data Streams
}

\author{
Rodrygo L. T. Santos \\ Univ. Federal de Minas Gerais \\ Belo Horizonte, MG, Brazil \\ rodrygo@dcc.ufmg.br \\ Ismail Sengor Altingovde \\ Middle East Technical University \\ Ankara, Turkey \\ altingovde@ceng.metu.edu.tr
}

\author{
Pablo Castells \\ Univ. Autónoma de Madrid \\ Madrid, Spain \\ pablo.castells@uam.es \\ Fazli Can \\ Bilkent University \\ Ankara, Turkey \\ canf@cs.bilkent.edu.tr
}

\begin{abstract}
This tutorial aims to provide a unifying account of current research on diversity and novelty in the domains of web search, recommender systems, and data stream processing.
\end{abstract}

\section{Categories and Subject Descriptors}

H.3.3 [Information Storage and Retrieval]: Information Search and Retrieval-search process

\section{Keywords}

Relevance, Diversity, Novelty, Ambiguity, Redundancy

\section{OVERVIEW}

Information retrieval (IR) is traditionally approached as a pursuit of relevant information, under the assumption that the users' information needs are unambiguously conveyed by their information requests. Arguably, such an assumption does not hold for the multitude of users' needs in modern IR systems, such as web search engines, recommender systems, and stream processing engines. In order to identify relevant information under the uncertainty posed by the users' requests, an effective approach is to diversify the retrieved results. By doing so, an IR system can minimize the chance of wrongly guessing the users' needs, which may cause the users to abandon their retrieval task.

Through a stream of active research and experiences, diversity and novelty can be said to have by now consolidated into a significant body of techniques, methodologies, theories, and knowledge in the field of information retrieval. This tutorial aims to provide a unifying account of current research on diversity and novelty in different IR domains. In particular, we will cover the motivations, as well as the most established approaches for producing and evaluating diverse

Permission to make digital or hard copies of part or all of this work for personal or classroom use is granted without fee provided that copies are not made or distributed for profit or commercial advantage, and that copies bear this notice and the full citation on the first page. Copyrights for third-party components of this work must be honored. For all other uses, contact the owner/author(s). Copyright is held by the author/owner(s).

WSDM'14, February 24-28, 2014, New York, New York, USA.

ACM 978-1-4503-2351-2/14/02.

http://dx.doi.org/10.1145/2556195.2556199. results in the context of search engines, recommender systems, and data streams. By contrasting the state-of the-art in these multiple domains, this tutorial aims to derive a common understanding of the diversification problem and the existing solutions, their commonalities and differences, as a means to foster new research directions.

In particular, the tutorial attendees will:

- understand the importance and complexities of achieving diversity/novelty for various IR domains;

- learn the state-of-the-art approaches for promoting diversity and novelty in web search, recommender systems, and data streams;

- learn the fundamental evaluation metrics and have an overview of past and current evaluation campaigns;

- get an overview of other related application areas that include query suggestions, query ambiguity detection, and aggregated search;

- obtain a unifying view of the topic by exploring the similarities and differences between the methods employed in different domains.

\section{TUTORIAL OUTLINE}

1. Practical and Theoretical Background

2. Diversity in Web Search

- Implicit and Explicit Diversification

- Advanced Topics in Web Search Diversification

- Diversity Evaluation

3. Diversity in Recommender Systems

- Motivation and Notions

- Novelty and Diversity Enhancement

- Novelty and Diversity Evaluation

4. Diversity in Data Streams

- Document-level Novelty

- Novelty and Diversification of Document Streams

- Evaluation

5. Other Application Areas 